\title{
Paradoxical Enhancement of Fear Extinction Memory and Synaptic Plasticity by Inhibition of the Histone Acetyltransferase p300
}

\author{
Roger Marek, ${ }^{1 \star}$ Carlos M. Coelho, ${ }^{1 \star}$ Robert K. P. Sullivan, ${ }^{1}$ Danay Baker-Andresen, ${ }^{1}$ Xiang Li, ${ }^{1}$ Vikram Ratnu, ${ }^{1}$ \\ Kevin J. Dudley, ${ }^{1}$ David Meyers, ${ }^{2}$ Chandrani Mukherjee, ${ }^{2}$ Philip A. Cole, ${ }^{2}$ Pankaj Sah, ${ }^{1}$ and Timothy W. Bredy ${ }^{1}$ \\ ${ }^{1}$ The University of Queensland, Queensland Brain Institute, Brisbane, Queensland 4072, Australia, and 2Department of Pharmacology and Molecular \\ Sciences, Johns Hopkins University School of Medicine, Baltimore, Maryland 21205
}

It is well established that the coordinated regulation of activity-dependent gene expression by the histone acetyltransferase (HAT) family of transcriptional coactivators is crucial for the formation of contextual fear and spatial memory, and for hippocampal synaptic plasticity. However, no studies have examined the role of this epigenetic mechanism within the infralimbic prefrontal cortex (ILPFC), an area of the brain that is essential for the formation and consolidation of fear extinction memory. Here we report that a postextinction training infusion of a combined p300/CBP inhibitor (Lys-CoA-Tat), directly into the ILPFC, enhances fear extinction memory in mice. Our results also demonstrate that the HAT p300 is highly expressed within pyramidal neurons of the ILPFC and that the small-molecule p300-specific inhibitor (C646) infused into the ILPFC immediately after weak extinction training enhances the consolidation of fear extinction memory. C646 infused $6 \mathrm{~h}$ after extinction had no effect on fear extinction memory, nor did an immediate postextinction training infusion into the prelimbic prefrontal cortex. Consistent with the behavioral findings, inhibition of p300 activity within the ILPFC facilitated long-term potentiation (LTP) under stimulation conditions that do not evoke long-lasting LTP. These data suggest that one function of p300 activity within the ILPFC is to constrain synaptic plasticity, and that a reduction in the function of this HAT is required for the formation of fear extinction memory.

\section{Introduction}

Substantial evidence indicates that extinction of conditioned fear, the reduction in responding to a feared cue when the cue is repeatedly presented without any adverse consequence, is new learning that inhibits the expression of a conditioned association rather than erasing it. The following three key findings underpin this conclusion: conditioned fear shows "spontaneous recovery" after the passage of time (Baum, 1988); "reinstatement" after presentations of the unconditioned stimulus alone (Rescorla and Heth, 1975); and "renewal" when the feared cue is presented in a context different from that of extinction training (Bouton and King, 1983). Efforts to understand the mechanisms of this form of learning have increased recently, particularly as it is an important model for the treatment of anxiety disorders. Like other forms of learning, long-lasting memory for fear extinction de-

Received Jan. 9, 2011; revised March 28, 2011; accepted April 1, 2011.

Author contributions:P.C., P.S., and T.W.B. designed research; R.M., C.M.C., R.K.P.S., D.B.-A., X.L., V.R., and K.J.D. performed research; R.M., C.M.C., R.K.P.S., D.M., C.M., P.A.C., P.S., and T.W.B. contributed unpublished reagents/ analytic tools; R.M., C.M.C., and T.W.B. analyzed data; R.M., C.M.C., P.A.C., P.S., and T.W.B. wrote the paper.

T.W.B. is supported by a generous grant from the Australian Research Council (DP1096148), P.S. is supported by a grant from the National Health and Medical Research Council (Grant APP569575), and D.M., C.M., and P.A.C. are supported by a grant from the National Institutes of Health (NIHGM62437). We would like to also thank Ms. Rowan Tweedale for helpful editing of the manuscript and Mr. Luke Hammond for assistance with confocal microscopy.

${ }^{*}$ R.M. and C.M.C. contributed equally to this work.

Correspondence should be addressed to Dr. Timothy W. Bredy, Queensland Brain Institute, The University of Queensland, Brisbane, QLD 4072, Australia. E-mail: t.bredy@uq.edu.au.

DOI:10.1523/JNEUROSCI.0133-11.2011

Copyright $\odot 2011$ the authors $\quad 0270-6474 / 11 / 317486-06 \$ 15.00 / 0$ pends on coordinated gene expression and the synthesis of new synaptic proteins (Martin et al., 2000; Bruel-Jungerman et al., 2007; Alberini, 2009). In recent years, it has been acknowledged that this process involves a tightly controlled interplay between transcriptional machinery and enzymes that regulate chromatin structure. Indeed, chromatin modification has been shown to play an essential role in the formation of long-term memory (Bredy et al., 2007, 2008; Barrett and Wood, 2008; Guan et al., 2009). These modifications include histone acetylation mediated by the histone acetyltransferase (HAT) and histone deacetylase (HDAC) families of transcriptional coactivators (Bayle and Crabtree, 1997). It is generally thought that histone acetylation is permissive for gene expression and that inhibition of HAT activity impairs, whereas inhibition of HDAC activity enhances, synaptic plasticity and memory (Sharma, 2010).

Activity of the HAT, p300, is critical for both object recognition and contextual fear memory but, interestingly, not for the acquisition of cued fear (Oliveira et al., 2007, 2011). These findings suggest that p300 is preferentially involved in the regulation of gene expression associated with hippocampus-dependent learning; however, it is not yet known whether p300 is functionally involved in establishing fear extinction memory. Given the important role of the HAT family of transcriptional coactivators in regulating gene expression associated with memory formation, and the fact that the formation of fear extinction memory depends on gene expression, protein synthesis, and synaptic plasticity in the infralimbic region of the medial prefrontal cortex 


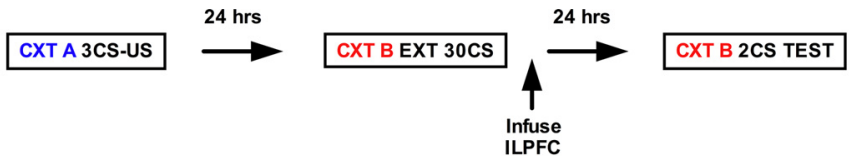

A

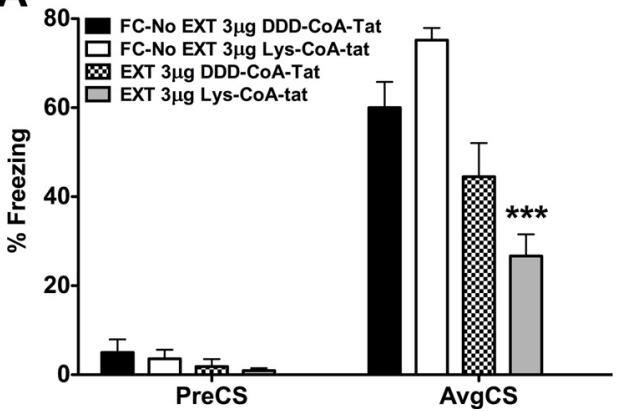

B

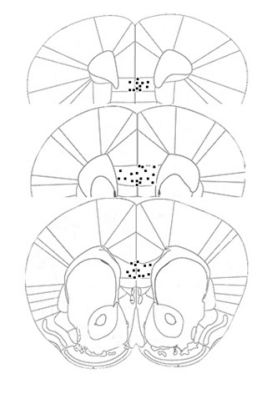

Figure 1. Combined inhibition of $\mathrm{p} 300 / \mathrm{CBP}$ in the infralimbic PFC enhances fear extinction memory. The experimental design is shown in the top. $A$, Relative to control mice (FC-No EXT, $3 \mu \mathrm{g}$;DDD-CoA-Tat, $n=12$ ), a postextinction training infusion of the combined $3300 / \mathrm{CBP}$ inhibitor Lys-CoA-Tat (EXT, $3 \mu \mathrm{g}, n=12$ ) directly into the ILPFC, enhances the formation of fear extinction memory. $\boldsymbol{B}$, Representative image of ILPFC cannula placement. Error bars, \pm SEM. preCS, Contextual freezing before tone exposure; AvgCS, average of two conditioned stimulus tone exposures at test. CTXA, Context A; $C$, conditioned stimulus; US, unconditioned stimulus; $C X T B$, context B. ${ }^{* * *} p<0.001$.
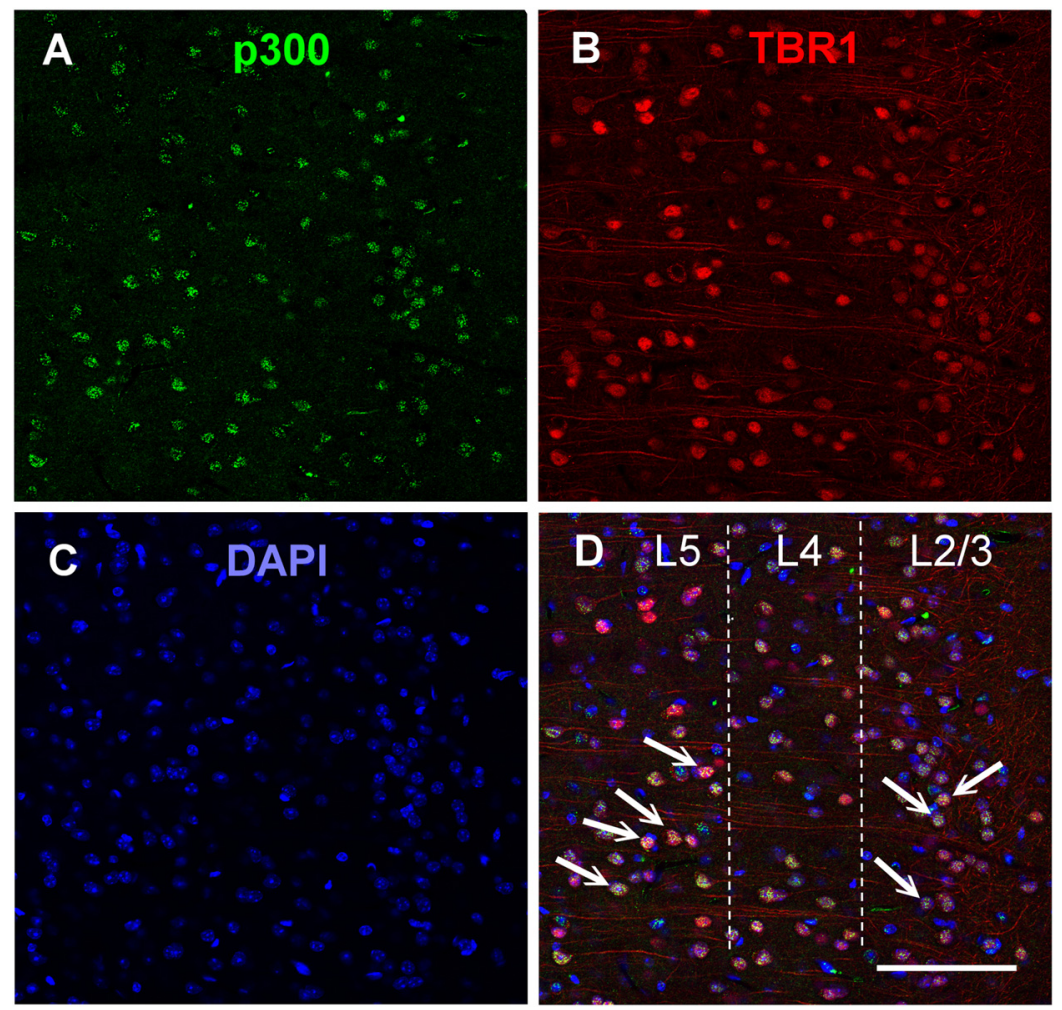

Figure 2. The histone acetyltransferase $\mathrm{p} 300$ is highly expressed within pyramidal neurons of the ILPFC. $A-D$, Immunohistochemical staining in layer $2 / 3$ (L2/3) and layer 5 (L5) of the ILPFC against 1300 (green) (A), the pyramidal neuron marker Tbr 1 (red) (B), DAPI (blue) (C), and merged (arrows indicate examples of colabeling) (D). Scale bar, $100 \mu \mathrm{m}$.

(ILPFC) (Milad and Quirk, 2002; Santini, 2004; Burgos-Robles et al., 2007; Laurent and Westbrook, 2009), we investigated whether p300 activity, within the ILPFC, is required for the formation of fear extinction memory. Immediately after fear extinction training, we microinfused a combined p300/cAMP-responsive elementbinding protein-binding protein (CBP) inhibitor (Lys-CoA-Tat), or a small-molecule p300-specific inhibitor (C646) directly into the ILPFC, then tested fear extinction memory $24 \mathrm{~h}$ later. In addition, to address the role of p300 in synaptic plasticity within the ILPFC, we induced long-term potentiation (LTP) within the ILPFC in the presence or absence of C646. Contrary to previous reports demonstrating a permissive role for HAT activation in memory and LTP, our results reveal that an inhibition of p300 within the ILPFC serves to strengthen fear extinction memory and enhance synaptic plasticity.

\section{Materials and Methods}

Mice. C57BL/6 male mice (8-10 weeks old) were housed four per cage, maintained on a $12 \mathrm{~h} \mathrm{light/dark}$ schedule, and allowed free access to food and water. All testing was conducted during the light phase in red light-illuminated testing rooms following protocols approved by the Institutional Animal Ethics Committee of the University of Queensland.

Immunohistochemistry. Whole brains from C57BL/6 mice $(n=4)$ were perfused and prepared for immunohistochemistry. Briefly, coronal sections (50 $\mu \mathrm{m}$ thick) were sliced on a vibratome (Leica) and blocked in 3\% bovine serum albumin (BSA) $/ 0.05 \%$ saponin for $1 \mathrm{~h}$. Sections were incubated in the primary antibodies p300 (Abcam; 1:2500) and tbr1 (Millipore; $1: 1000)$ prepared in blocking solution $(0.5 \%$ BSA, $0.05 \%$ saponin, $0.05 \%$ sodium azide) for $56 \mathrm{~h}$ at room temperature. Sections were washed in PBS and then incubated in fluorescently tagged secondary antibodies (anti-rabbit IgG-Alexa-488 or anti-mouse IgG-Alexa-555 (1:1000, Invitrogen) for $5 \mathrm{~h}$. Sections were washed in PBS and then slide mounted with $50 \%$ glycerol/ PBS and 4', 6' -diamidino-2-phenylindole dihydrochloride (DAPI). Sections were viewed and captured using a Zeiss confocal fluorescence microscope.

Drugs. The combined p300/CBP inhibitor Lys-CoA-Tat and its inactive analog DDDCoA-Tat, and the small molecule p300-specific inhibitor C646 and its inactive analog C37 were synthesized and purified as described by Lau et al. (2000) and Bowers et al. (2010). The specificity of HAT inhibition by Lys-CoA-Tat and C646 was determined previously by spectrophotometric HAT assay (Guidez et al., 2005; Min et al., 2010). Furthermore, Min et al. (2010) have shown that the inhibitory effect of C646 on p300-mediated acetylation is mimicked by a siRNA designed to knock down p300. The control compound C37 differs from C646 by only one double bond but is completely silent as a p300 inhibitor, therefore, controlling for any off-target effects of C646 (Bowers et al., 2010). In the behavioral experiments described below, all mice were infused with drug directly into either the ILPFC or, in control experiments, into the prelimbic PFC (PLPFC).

Surgery. Double cannulae (Plastics One) were implanted into the IL$\mathrm{PFC}$ or the PLPFC along the midline in the anterior posterior (AP) plane, a minimum of $3 \mathrm{~d}$ before behavioral training. ILPFC cannulae were centered at AP $+1.78 \mathrm{~mm}$, and dorsoventral (DV) $-2.9 \mathrm{~mm}$. For the PLPFC infusion experiments, cannulae were centered at AP $+1.78 \mathrm{~mm}$ and DV $-1.9 \mathrm{~mm}$. After behavioral testing, all mice were transcardially perfused and their brains dissected, sectioned, and Nissl stained (1:1000) to confirm placement of the cannulae. 
Behavioral experiments. For extinction of conditioned fear, cued fear was induced in naive mice with three pairings of a $2 \mathrm{~min}, 80 \mathrm{~dB}$, white noise conditioned stimulus (CS) coterminating with a $1 \mathrm{~s}, 0.7 \mathrm{~mA}$ footshock [2 min interstimuli interval (ISI)]. Mice were matched into equivalent treatment groups based on freezing during the third training CS. Twenty-four hours later, mice were placed in context B and allowed to acclimate for $2 \mathrm{~min}$. For the p300/CBP experiment, extinction training consisted of 30 nonreinforced $2 \mathrm{~min}$ CS presentations ( $5 \mathrm{~s}$ ISI). In this experiment, a 30 CS partial extinction training protocol was used to test whether a drug manipulation led to either impairment or enhancement of fear extinction memory (Cain et al., 2004). In experiments examining the effect of p300-specific inhibition by $\mathrm{C} 646$ on fear extinction memory, extinction training consisted of 5 nonreinforced 2 min CS presentations (5 s ISI). A 5 CS protocol allows the direct examination of the effect of a drug on the enhancement of extinction memory because this training protocol does not lead to persistent extinction (Cain et al., 2004). In the first experiment, immediately following the last CS exposure, mice were infused directly into the ILPFC with the combined p300/CBP inhibitor Lys-CoA-Tat (or its control DDD-COA-Tat, $2 \times 0.75 \mu \mathrm{l}$ injection volume, $1.5 \mu \mathrm{g}$, administered over $2 \mathrm{~min}$ ). In subsequent experiments, immediately following the last CS exposure, mice were infused directly into the ILPFC with either the p300 inhibitor (C646) or its silent analog (C37) (2X $0.75 \mu \mathrm{l}$ injection volume in each case, $1.5 \mu \mathrm{g}$, administered over $2 \mathrm{~min}$ ). As a control in each experiment, fear-conditioned mice without extinction (FC-No EXT) were placed in context $\mathrm{B}$ for a time equal to that of the extinctionlearning group, and drug was subsequently infused directly into the ILPFC immediately after context exposure. In all experiments, freezing was assessed $24 \mathrm{~h}$ later during two 2 min CS presentations ( 2 min ISI) in context B. For fear acquisition, mice were infused with $\mathrm{C} 646$ or C37 directly into the PLPFC immediately after cued-fear training (as described) and tested in context B $24 \mathrm{~h}$ later. The effect of C646 infused into the ILPFC $6 \mathrm{~h}$ after extinction training was also examined to control for any effect of p300 inhibition that may occur outside the window for consolidation of fear extinction memory. Finally, to control for the regional specificity of the effect of $\mathrm{C} 646$ on fear extinction, a separate group of mice was infused with C646 or C37, directly into the PLPFC, immediately after extinction training and tested the following day. As a measure of memory strength, behavioral freezing, the absence of all nonrespiratory movements, was automatically rated during all phases in conditioning chambers (Coulburn Instruments) by digital cameras mounted in the ceiling of each chamber and connected via a quad processor (Freezeframe). The percentage of freezing was calculated for each mouse, and data were represented as mean \pm SEM freezing percentages for groups of mice during specified time bins (Prism, GraphPad). Total session means were analyzed by one-way ANOVA with Dunnetts post hoc test (all groups relative to FC-No EXT control for the fear extinction experiments and No Shock control for the fear acquisition experiment).

Electrophysiology. C57BL/6 adult mice (age range, 24-33 d) were anesthetized with isoflurane and killed by decapitation. Brains were rapidly removed and placed in ice-cold artificial CSF ( $\mathrm{aCSF}$ ) containing the following (in mM): $\mathrm{NaCl} 118, \mathrm{KCl} 2.5, \mathrm{NaHCO}_{3} 25$, glucose $10, \mathrm{MgCl}_{2} 1.3$, $\mathrm{CaCl}_{2}$ 2.5, and $\mathrm{NaH}_{2} \mathrm{PO}_{4}$ 1.2. The 300- $\mu$ m-thick coronal brain slices containing the medial PFC were prepared with a vibratome (VT 1000S, Leica). Slices were allowed to recover in oxygenated $\left(95 \% \mathrm{O}_{2} / 5 \% \mathrm{CO}_{2}\right)$ aCSF at $35^{\circ} \mathrm{C}$ for at least $30 \mathrm{~min}$, then were kept at room temperature for at least another $30 \mathrm{~min}$ before experiments were commenced. Slices were transferred to the recording chamber as required and were continuously perfused with oxygenated aCSF through a gravity-fed system while being maintained at $30-32^{\circ} \mathrm{C}$. The perfusate (either $25 \mathrm{ml}$ aCSF or $25 \mathrm{~nm} \mathrm{C} 646$ in $25 \mathrm{ml}$ of aCSF) was recycled using a peristaltic pump to reduce drug usage (Econo Pump EP-1). Experiments were performed in the presence of picrotoxin $(100 \mu \mathrm{M})$ and CGP-35348 $(1 \mu \mathrm{M})$ to block GABAergic transmission. Field responses were recorded from layer 5 using glass electrodes filled with $3 \mathrm{M} \mathrm{NaCl}$ (pipette resistance: 3-4 M $\Omega$ ) and a bipolar stimulating electrode was placed in layer $2 / 3$. Synaptic responses were evoked at $0.1 \mathrm{~Hz}$, and LTP was induced using one of two high-frequency stimulation (HFS) protocols. For strong LTP induction, 5 highfrequency trains ( 5 pulses at $100 \mathrm{~Hz}$ ) were repeated 20 times with an interpulse interval of $200 \mathrm{~ms}$ repeated 5 times ( $6 \mathrm{~s}$ between trains). The weak induction protocol consisted of trains of 5 stimulations at $50 \mathrm{~Hz}$, repeated 10 times with an intertrain interval of $4 \mathrm{~s}$. Effects of HFS were calculated by averaging $10 \mathrm{~min}$ (60 sweeps) of recordings immediately before and $60 \mathrm{~min}$ after LTP induction. Signals were recorded using a patch-clamp amplifier (Multiclamp 700B, Molecular Devices). Responses were filtered at $4-8 \mathrm{kHz}$ and digitized at $10 \mathrm{kHz}$ (ITC-16, In- 


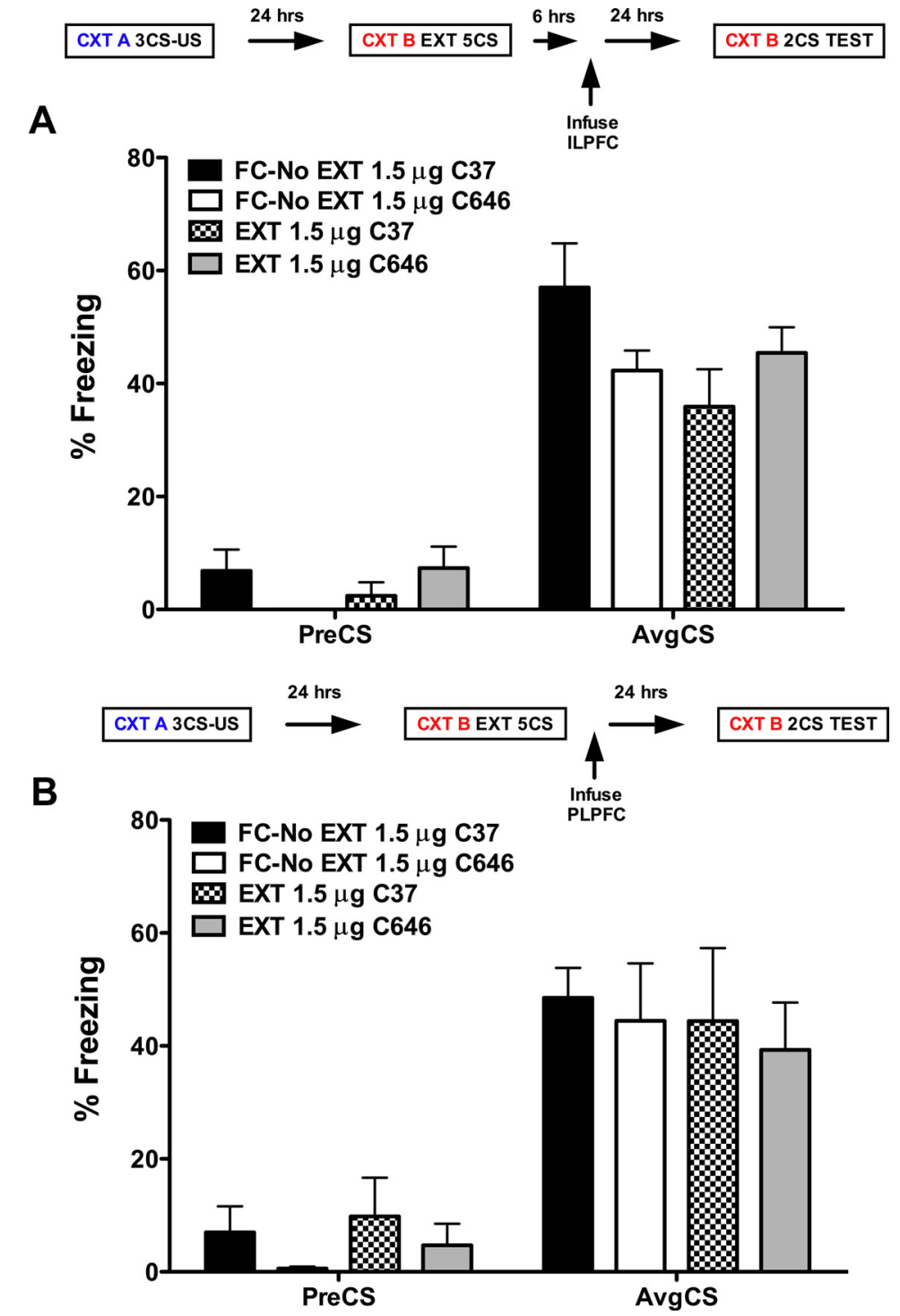

Figure 4. $\boldsymbol{A}$, Six hours after extinction training, infusion of the p300 inhibitor (646 (EXT, $1.5 \mu \mathrm{g}, n=7$ ), directly into the infralimbic prefrontal cortex (ILPFC), has no effect on the formation of fear extinction memory. $\boldsymbol{B}$, An immediate postextinction training infusion of the $\mathrm{p} 300$ inhibitor (646 (EXT, $1.5 \mu \mathrm{g}, n=7$ ), directly into the prelimbic prefrontal cortex (PLPFC), has no effect on the formation of fear extinction memory.

strutech). All data were acquired, stored, and analyzed on a Macintosh using Axograph X (version 1.2.1, Axograph). A repeatedmeasures ANOVA was used for statistical comparisons between groups. Results are expressed as mean \pm SEM.

\section{Results}

Enhanced fear extinction memory after immediate postextinction training infusion of the combined p300/CBP inhibitor Lys-CoA-Tat

In our initial experiments, we tested the role of the HAT p300/CBP in the consolidation of fear extinction memory. Animals underwent cued fear conditioning on day 1 and fear extinguished in a different context $24 \mathrm{~h}$ later (Fig. 1). The combined p300/CBP inhibitor LysCoA-Tat or its control (DDD-CoA-Tat) was infused into the ILPFC immediately after extinction training (30 CS). Relative to retention control mice (FC-No EXT DDD-CoA-Tat), the 30 CS extinction training protocol did not lead to persistent extinction memory in extinction-trained control mice (EXT DDD-CoA-Tat). However, there was a significant reduction in freezing in mice that had received
Lys-CoA-Tat immediately after extinction training $\left(F_{(3,42)}=15.73\right.$; $p<0.001$; Dunnett's post hoc test, FC-No EXT DDD-CoATat vs EXT Lys-CoA-Tat, $p<0.0001$ ) (Fig. $1 A)$, showing enhanced extinction memory when tested $24 \mathrm{~h}$ after drug treatment. These findings prompted us to carry out a more detailed examination of the effect of specifically inhibiting p300 on the consolidation of fear extinction memory and synaptic plasticity in the ILPFC.

\section{Enhanced fear extinction memory after immediate postextinction training infusion of C646}

Immunohistochemical labeling revealed that p300 was expressed in the nuclei of neurons stained for DAPI and that p300 expression was coexpressed in pyramidal neurons labeled for tbr1 in layer $2 / 3$ and layer 5 of the ILPFC (Fig. 2). To examine the role of p300 in the ILPFC during the formation of fear extinction memory, a small-molecule inhibitor specific for C646, or a control compound (C37) was infused into the ILPFC immediately after extinction training (5 CS). Relative to retention control mice (FC-No EXT C37), the 5CS extinction training protocol did not lead to persistent extinction memory in extinction-trained control mice (EXT C37). However, there was a significant reduction in freezing in mice that had received C646 immediately after extinction training $\left(F_{(3,23)}=4.70 ; p<0.05\right.$; Dunnett's post hoc test, FC- No EXT C37 vs EXT C646, $p<0.05$ ) (Fig. 3A). During fear acquisition, although all mice learned to freeze in response to the tone relative to the no-shock controls, there was no effect of PLPFC infusion of C646 on memory for cued fear (Fig. $3 B$ ). There was also no effect of C646 on fear extinction memory if the compound was infused $6 \mathrm{~h}$ after extinction training (Fig. 4A) or if C646 was infused into the PLPFC immediately after fear extinction training (Fig. $4 B$ ).

p300 inhibition facilitates LTP induced by weak tetanic stimulation

To explore the mechanism underlying the effect of p300 inhibition on the formation of fear extinction memory, we tested its actions on LTP in the ILPFC. Stimulation of layer $2 / 3$ evoked a stable field potential in layer 5 (Fig. $5 \mathrm{~A}$ ) that was abolished by the AMPA/kainate receptor antagonist NBQX $(10 \mu \mathrm{M})$ (Fig. 5D). Tetanic stimulation using a strong HFS protocol led to stable LTP $\left(F_{(1,9)}=2.93, p<0.01 ; 120 \pm 0.9 \%, n=5\right.$ /group) (Fig. $\left.5 B\right)$, whereas in control slices a weak stimulation protocol led to a small and variable potentiation of the field potential that returned to baseline by $30 \min \left(F_{(1,9)}=0.42, p>0.05 ; 98.2 \pm 1.2 \%\right.$, $n=3-4$ /group) (Fig. 5E). Application of C646 had no effect on LTP induced by the strong HFS protocol (Fig. $5 B, C$ ); however, delivery of weak tetanic stimulation in the presence of C646- 
induced LTP, which persisted for at least $60 \min \left(F_{(1,9)}=7.43, p=0.041 ; 113.7 \pm\right.$ $0.8 \%, n=5$ ) (Fig. $5 E, F$ ).

\section{Discussion}

In this study, we report two novel findings: (1) inhibition of p300 activity in the ILPFC enhances the formation of fear extinction memory in mice; and (2) p300 inhibition enhances LTP in ILPFC pyramidal neurons under conditions that do not normally evoke a persistent effect on excitatory transmission. These findings suggest that one possible function of p300 within the ILPFC is to constrain synaptic plasticity, and a reduction in the activity of this HAT is required for the formation of fear extinction memory.

A role for p300 in learning and memory was established in two earlier reports. Transgenic mice with a truncated form of p300 (Oliveira et al., 2007) and mice with a forebrain-specific conditional deletion of p300 (Oliveira et al., 2011) both show impaired hippocampus-dependent memory formation. In agreement with the findings of Oliveira et al. $(2007,2011)$, we also observed no effect of p300 inhibition on the acquisition of cued fear (Fig. 3B). We tested the effect of a combined p300/CBP inhibitor, Lys-CoA-Tat, on the formation of fear extinction memory using a partial extinction protocol (30 CS), which elicits moderate fear extinction that is sensitive to either disruption or enhancement (Cain et al., 2004, Bredy and Barad, 2008). Contrary to previous studies demonstrating an impairment in learning and memory after p300 or CBP knockdown, we observed a significant enhancement of fear extinction memory when the mice were tested $24 \mathrm{~h}$ after an immediate postextinction training infusion of the combined p300/CBP inhibitor Lys-CoA-Tat (Fig. 1). These initial findings prompted us to explore the role of $\mathrm{p} 300$ in fear extinction in more detail by testing the effect of the small molecule p300 inhibitor C646 on fear extinction memory using a weak extinction protocol that does not generate a persistent extinction memory (5 CS) (Cain et al., 2004). Again, we observed a significant enhancement of fear extinction memory when the mice were tested $24 \mathrm{~h}$ after an immediate postextinction training infusion of C646 (Fig. 3A). Importantly, these effects were not observed if the C646 was infused $6 \mathrm{~h}$ after extinction training (Fig. $4 A$ ), nor were they observed if the drug was infused into the PLPFC immediately after extinction training (Fig. 4B). In accordance with the effect of C646 on fear extinction memory after weak extinction training, C646 also facilitated LTP within the ILPFC. Using an LTP protocol similar to one that has previously demonstrated a necessary role for the HAT CBP in hippocampal LTP (Vecsey et al., 2007), inhibition of p300 facilitated the induction of long-lasting LTP. Indeed, a weak stimulation protocol that did not induce LTP was able to do so in the presence of the p300 inhibitor (Fig. 5C). Together, these findings suggest an antagonistic role for p300 in the formation of fear extinction memory and in synaptic plasticity within the ILPFC.

These paradoxical observations raise an important question: how does p300 activity within the ILPFC influence extinction memory and synaptic plasticity? HAT activity has mainly been
B

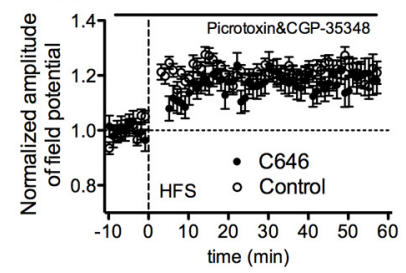

C

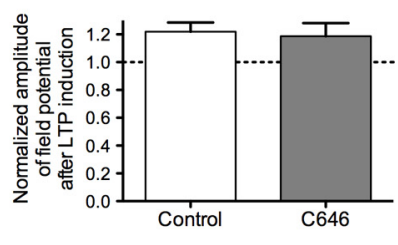

E

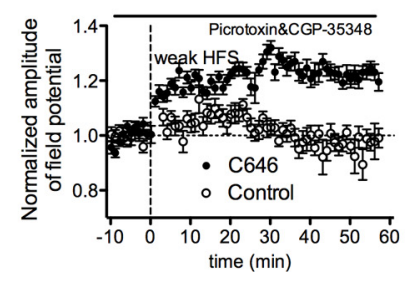

$\mathbf{F}$

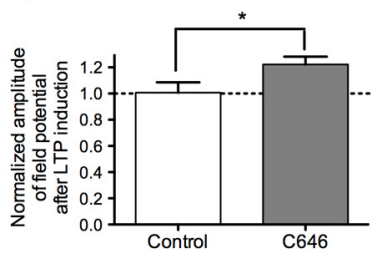

Figure 5. Inhibition of $\mathrm{p} 300$ enhances LTP in the infralimbic PFC. $A$, LTP induction in coronal ILPFC slices by electric stimulation of a $2 / 3$ and recording of a field potential from layer $5 . B$, Application of strong high-frequency stimulation $(100 \mathrm{~Hz})$ to layer $2 / 3$ induction (average over $10 \mathrm{~min}$ ) reveals no significant difference between control and (646-treated slices. $\boldsymbol{D}$, Field potential to weaker $50 \mathrm{~Hz}$ stimulation protocol to layer 2/3 neurons in the presence of GABA antagonists led to LTP induction in only 6 (646-

associated with enhanced LTP induction (Alarcón et al., 2004) and memory formation (Yeh et al., 2004; Vecsey et al., 2007; Maurice et al., 2008). p300, originally described as a transcriptional coactivator, interacts with many proteins, including nuclear factor $\kappa \mathrm{B}(\mathrm{NF}-\kappa \mathrm{B})$, yin-yang 1 (YY1), p53, and activating transcription factor 2 (Perkins et al., 1997; Yao et al., 2001; Furia et al., 2002; Dornan et al., 2003; Karanam et al., 2007). The interaction with NF- $\kappa$ B and YY1 are interesting given that they have been shown to play important roles in the reconsolidation of fear and in hippocampus-dependent learning and memory (Lubin and Sweatt, 2007; Gao et al., 2010). In particular, NF- $\kappa$ B levels are tightly regulated in memory consolidation and extinction, depending on the strength of re-exposure to the training context (Merlo and Romano, 2008), suggesting that memory reconsolidation requires $\mathrm{NF}-\kappa \mathrm{B}$-dependent regulation of gene expression. Since p300 is involved in the NF- $\kappa \mathrm{B}$ signaling pathway (Quivy and Van Lint, 2004; Calao et al., 2008), its inhibition at the time of extinction memory consolidation might alter NF- $\kappa$ B levels, resulting in a disruption of fear memory reconsolidation and therefore an enhancement of fear extinction, in a similar way to that observed by Merlo and Romano (2008).

Although p300 can acetylate cytoplasmic proteins (Min et al., 2010), it also interacts with transcriptional repressors such as YY1 (Lee et al., 1995; Thomas and Seto, 1999). An augmented repressor activity of YY1 by p300 coactivation at residues 170-200 was observed by Yao et al. (2001), suggesting that inhibition of p300 binding to YY1 reduces the latter's repressive function, thereby leading to increased transcriptional activity of target genes [e.g., brain-derived neurotrophic factor (BDNF)] and enhanced synaptic plasticity. In support of this hypothesis, a loss-of-function mutation of SIRT1, which acts via YY1, reduces BDNF expression, and results in impaired novel object recognition and decreased LTP in the CA1 region of the hippocampus (Gao et al., 2010). Our data suggest that a potential role for a p300-YY1 interaction in the ILPFC is to constrain plasticity, whereby a re- 
duction in p300 activity may reduce the negative regulatory influence of YY1, leading to increased BDNF expression under weak extinction training conditions. This would subsequently result in enhanced fear extinction memory and the facilitation of LTP.

Our findings reveal a complex relationship between epigenetic mechanisms and the consolidation of long-term memory. The paradoxical enhancement of fear extinction memory and LTP by inhibition of p300 activity within the ILPFC suggests that, under certain conditions, rather than playing a permissive role in memory formation, transcriptional coactivators such as the HAT p300 may serve to constrain learning-related plasticity.

\section{References}

Alarcón JM, Malleret G, Touzani K, Vronskaya S, Ishii S, Kandel ER, Barco A (2004) Chromatin acetylation, memory, and LTP are impaired in CBP $+/-$ mice: a model for the cognitive deficit in Rubinstein-Taybi syndrome and its amelioration. Neuron 42:947-959.

Alberini CM (2009) Transcription factors in long-term memory and synaptic plasticity. Physiol Rev 89:121-145.

Barrett RM, Wood MA (2008) Beyond transcription factors: the role of chromatin modifying enzymes in regulating transcription required for memory. Learn Mem 15:460-467.

Baum M (1988) Spontaneous recovery from the effects of flooding (exposure) in animals. Behav Res Ther 26:185-186.

Bayle JH, Crabtree GR (1997) Protein acetylation: more than chromatin modification to regulate transcription. Chem Biol 4:885-888.

Bouton ME, King DA (1983) Contextual control of the extinction of conditioned fear: tests for the associative value of the context. J Exp Psychol Anim Behav Process 9:248-265.

Bowers EM, Yan G, Mukherjee C, Orry A, Wang L, Holbert MA, Crump NT, Hazzalin CA, Liszczak G, Yuan H, Larocca C, Saldanha SA, Abagyan R, Sun Y, Meyers DJ, Marmorstein R, Mahadevan LC, Alani RM, Cole PA (2010) Virtual ligand screening of the p300/CBP histone acetyltransferase: identification of a selective small molecule inhibitor. Chem Biol 17:471-482.

Bredy TW, Barad M (2008) The histone deacetylase inhibitor valproic acid enhances acquisition, extinction, and reconsolidation of conditioned fear. Learn Mem 15:39-45.

Bredy TW, Wu H, Crego C, Zellhoefer J, Sun YE, Barad M (2007) Histone modifications around individual BDNF gene promoters in prefrontal cortex are associated with extinction of conditioned fear. Learn Mem 14:268-276.

Bruel-Jungerman E, Davis S, Laroche S (2007) Brain plasticity mechanisms and memory: a party of four. Neuroscientist 13:492-505.

Burgos-Robles A, Vidal-Gonzalez I, Santini E, Quirk GJ (2007) Consolidation of fear extinction requires NMDA receptor-dependent bursting in the ventromedial prefrontal cortex. Neuron 53:871-880.

Cain CK, Blouin AM, Barad M (2004) Adrenergic transmission facilitates extinction of conditional fear in mice. Learn Mem 11:179-187.

Calao M, Burny A, Quivy V, Dekoninck A, Van Lint C (2008) A pervasive role of histone acetyltransferases and deacetylases in an NF- $\kappa$ B-signaling code. Trends Biochem Sci 33:339-349.

Dornan D, Shimizu H, Perkins ND, Hupp TR (2003) DNA-dependent acetylation of p53 by the transcription coactivator p300. J Biol Chem 278:13431-13441.

Furia B, Deng L, Wu K, Baylor S, Kehn K, Li H, Donnelly R, Coleman T, Kashanchi F (2002) Enhancement of nuclear factor $-\kappa \mathrm{B}$ acetylation by coactivator p300 and HIV-1 tat proteins. J Biol Chem 277:4973-4980.

Gao J, Wang WY, Mao YW, Gräff J, Guan JS, Pan L, Mak G, Kim D, Su SC, Tsai LH (2010) A novel pathway regulates memory and plasticity via SIRT1 and miR-134. Nature 466:1105-1109.

Guan JS, Haggarty SJ, Giacometti E, Dannenberg JH, Joseph N, Gao J, Nieland TJ, Zhou Y, Wang X, Mazitschek R, Bradner JE, DePinho RA, Jaenisch R, Tsai LH (2009) HDAC2 negatively regulates memory formation and synaptic plasticity. Nature 459:55-60.
Guidez F, Howell L, Isalan M, Cebrat M, Alani RM, Ivins S, Hormaeche I, McConnell MJ, Pierce S, Cole PA, Licht J, Zelent A (2005) Histone acetyltransferase activity of $\mathrm{p} 300$ is required for transcriptional repression by the promyelocytic leukemia zinc finger protein. Mol Cell Biol 25:5552-5566.

Karanam B, Wang L, Wang D, Liu X, Marmorstein R, Cotter R, Cole PA (2007) Multiple roles for acetylation in the interaction of p300 HAT with ATF-2. Biochemistry 46:8207-8216.

Lau OD, Kundu TK, Soccio RE, Ait-Si-Ali S, Khalil EM, Vassilev A, Wolffe AP, Nakatani Y, Roeder RG, Cole PA (2000) HATs off: selective synthetic inhibitors of the histone acetyltransferases p300 and PCAF. Mol Cell 5:589-595.

Laurent V, Westbrook RF (2009) Inactivation of the infralimbic but not the prelimbic cortex impairs consolidation and retrieval of fear extinction. Learn Mem 16:520-529.

Lee JS, Galvin KM, See RH, Eckner R, Livingston D, Moran E, Shi Y (1995) Relief of YY1 transcriptional repression by adenovirus E1A is mediated by E1A-associated protein p300. Gene Dev 9:1188-1198.

Lubin FD, Sweatt JD (2007) The IkB kinase regulates chromatin structure during reconsolidation of conditioned fear memories. Neuron 55: 942-957.

Martin SJ, Grimwood PD, Morris RG (2000) Synaptic plasticity and memory: an evaluation of the hypothesis. Annu Rev Neurosci 23:649-711.

Maurice T, Duclot F, Meunier J, Naert G, Givalois L, Meffre J, Célérier A, Jacquet C, Copois V, Mechti N, Ozato K, Gongora C (2008) Altered memory capacities and response to stress in p300/CBP-associated factor (PCAF) histone acetylase knockout mice. Neuropsychopharmacology 33:1584-1602.

Merlo E, Romano A (2008) Memory extinction entails the inhibition of the transcription factor NF- $\kappa$ B. PLoS One 3:e3687.

Milad MR, Quirk GJ (2002) Neurons in medial prefrontal cortex signal memory for fear extinction. Nature 420:70-74.

Min SW, Cho SH, Zhou Y, Schroeder S, Haroutunian V, Seeley WW, Huang EJ, Shen Y, Masliah E, Mukherjee C, Meyers D, Cole PA, Ott M, Gan L (2010) Acetylation of tau inhibits its degradation and contributes to tauopathy. Neuron 67:953-966.

Oliveira AM, Wood MA, McDonough CB, Abel T (2007) Transgenic mice expressing an inhibitory truncated form of $\mathrm{p} 300$ exhibit long-term memory deficits. Learn Mem 14:564-572.

Oliveira AM, Estévez MA, Hawk JD, Grimes S, Brindle PK, Abel T (2011) Subregion-specific p300 conditional knock-out mice exhibit long-term memory impairments. Learn Mem 18:161-169.

Perkins ND, Felzien LK, Betts JC, Leung K, Beach DH, Nabel GJ (1997) Regulation of NF- $\kappa$ B by cyclin-dependent kinases associated with the p300 coactivator. Science 275:523-527.

Quivy V, Van Lint C (2004) Regulation at multiple levels of NF-kBmediated transactivation by protein acetylation. Biochem Pharmacol 68:1221-1229.

Rescorla RA, Heth CD (1975) Reinstatement of fear to an extinguished conditioned stimulus. J Exp Psychol Anim Behav Process 1:88-96.

Santini E, Ge H, Ren K, Peña de Ortiz S, Quirk GJ (2004) Consolidation of fear extinction requires protein synthesis in the medial prefrontal cortex. J Neurosci 24:5704-5710.

Sharma SK (2010) Protein acetylation in synaptic plasticity and memory. Neurosci Biobehav Rev 34:1234-1240.

Thomas MJ, Seto E (1999) Unlocking the mechanisms of transcription factor YY1: are chromatin modifying enzymes the key? Gene 236:197-208.

Vecsey CG, Hawk JD, Lattal KM, Stein JM, Fabian SA, Attner MA, Cabrera SM, McDonough CB, Brindle PK, Abel T, Wood MA (2007) Histone deacetylase inhibitors enhance memory and synaptic plasticity via CREB: CBP-dependent transcriptional activation. J Neurosci 27:6128-6140.

Yao YL, Yang WM, Seto E (2001) Regulation of transcription factor YY1 by acetylation and deacetylation. Mol Cell Biol 21:5979-5991.

Yeh SH, Lin CH, Gean PW (2004) Acetylation of nuclear factor-kB in rat amygdala improves long-term but not short-term retention of fear memory. Mol Pharmacol 65:1286-1292. 